\title{
Image Enhancement by Using Directional Wavelet Transform
}

\author{
D. Heric, B. Potocnik \\ University of Maribor, Faculty of Electrical Engineering and Computer Science, Slovenia
}

\begin{abstract}
The purpose of this paper is to introduce a novel image enhancement technique by using directional wavelet transform. Directional wavelet transform decomposes an image into four-dimensional space which augments the image by the scale and directional information. We show that the directional information significantly improves image enhancement in noisy images in comparison with the classical techniques. Image enhancement is based on the multiscale singularity detection with an adaptive threshold whose value is calculated via maximum entropy measure. The proposed technique was tested on synthetic images at different signal-to-noise ratios and clinical images.
\end{abstract}

Keywords: image enhancement, wavelet transform, directional wavelet transform.

\section{Introduction}

Image enhancement is a technique which reduces image noise, removes artefacts, and preserves details. Its purpose is to amplify certain image features for analysis, diagnosis and display. The technique can be performed by either suppressing the noise or increasing the image contrast [1]. A comprehensive survey of enhancement methods has been published in [2].

Elementary enhancement techniques are histogram-based. They are simple, fast, and with them acceptable results for some applications can be achieved. But their biggest disadvantages are limited ability to incorporate local context into the transformation. Afterward, an adaptive histogram equalization technique has been proposed [3], which brings a limited improvement, because fixed contextual regions cannot adapt to features of different size. In an attempt to overcome these limitations, another, more advanced and most widespread enhancement algorithm has been proposed which has a common strategy-edge sharpening.

The technique of unsharp masking has become a popular enhancement to assist in diagnosis [3]. Unsharp masking sharpens the edges by subtracting a portion of a filtered component from an original image [4]. An extension of this technique is the Laplacian filtering proposed in [5]. However, techniques of unsharp masking are less efficient for images containing a wide range of features because of their singlescale properties. Refinements were introduced in $[6,7,8]$ with novel local contrast measure and nonlinear transform function. Their major drawback is the absence of explicit noise suppression model that can cause aplification of the noise or artefacts.

An advancement of wavelet theory has taken the interest of researchers in its application to image enhancement $[9,10,11,12]$. In $[11]$ the enhancement is done by noise removing and edge enhancement. The algorithm relies on a multiscale edge representation where the noise is connected to the singularities. In [12] the nonlinear wavelet shrinkage algorithm is proposed which reduces wavelet coefficients toward zero, based on a level-dependent threshold. In this work, we give a detailed mathematical analysis of a directional wavelet transform and reveal its connection with the edge enhancement. In addition, we discuss a single level edge sharpening, followed by its refinement to a multiscale sharpening.

This paper is organized as follows: Section 2 describes a directional wavelet transform. Sec- 
tion 3 introduces a single scale edge sharpening method. Section 4 deals with image enhancement. Section 5 presents some experimental results and compares with Laplacian sharpening. Finally, Section 6 concludes this work and suggests possible future research directions.

\section{Directional Wavelet Transform}

Natural images are not simple stacks of 1-D piecewise smooth scan-lines; discontinuity points (i.e. edges) are typically located along smooth curves (i.e. contours) owing to smooth boundaries of physical objects. Thus, natural images contain intrinsic geometrical structures that are key features in visual information [13]. In order to identify such structures, their orientation bears a crucial piece of information.

The wavelet transform [14] has a long and successful history as an efficient image processing tool. However, as a result of a separable extension from 1-D bases, wavelets in higher dimensions can only capture very limited directional information. Different directions are mixed in certain wavelet subbands. For instance, 2-D wavelets only provide three directional components, namely horizontal, vertical, and diagonal. Furthermore, the $45^{\circ}$ and $135^{\circ}$ directions are mixed in diagonal subbands. With the directional extension for wavelets proposed in [15] that mixing problem can be improved, but not totally resolved. However, the wavelet transform is still very attractive for image processing applications.

A number of approaches in providing finer directional decomposition have been proposed. Some notable examples include 2-D Gabor wavelets, the steerable pyramid, the directional filter bank, 2-D directional wavelets, complex wavelets, curvelets [16], ridgelets [16], and contourlets [13]. Among all, we decided to use directional wavelet transform [17] because it retains all beneficial properties of wavelets and provides additional directional information at the same time.

The directional wavelet transform (DWT) of discrete image $\mathrm{I}(\mathrm{m}, \mathrm{n})$ is defined by $(1)$

$$
\begin{array}{r}
K_{s}\left(\alpha, \tau_{1}, \tau_{1} \delta\right) \\
=\sum_{m=0}^{M-1} \sum_{n=0}^{N-1} \frac{1}{\sqrt{\alpha}} I(m, n) Q(\delta) . \\
\cdot \kappa_{\alpha}\left(\tau_{1}-m, \tau_{2}-n\right)
\end{array}
$$

where $\alpha$ is the scale parameter, $\kappa_{\alpha}(m, n)$ stands for 2-D mother wavelet kernel, while $M$ and $N$ represent the kernel's size. Expression $Q(\delta)$ is the rotator which rotates kernel $\kappa_{\alpha}$ counterclockwise by an angle $\delta$. Kernel $\kappa_{\alpha}$ is defined by (2)

$$
\kappa_{\alpha}(m, n)=\psi_{\alpha}(n), \quad m \in[0, M-1] .
$$

In the sequel, we derive the algorithm for the directional wavelet transform. As a wavelet function we decided to use the 1-D Mexican Hat function defined by

$$
\psi(t)=\frac{2}{\pi^{1 / 4} \sqrt{3 \sigma}}\left(\frac{t^{2}}{\sigma^{2}}-1\right) e^{\left(\frac{-t^{2}}{2 \sigma^{2}}\right)} .
$$

Mexican Hat is equal to the second derivative of a Gaussian and it was widely used in computer vision to detect multiscale edges $[18,19]$. Thus, we use 2-D Mexican Hat kernel whose sample is depicted in Figure 1.

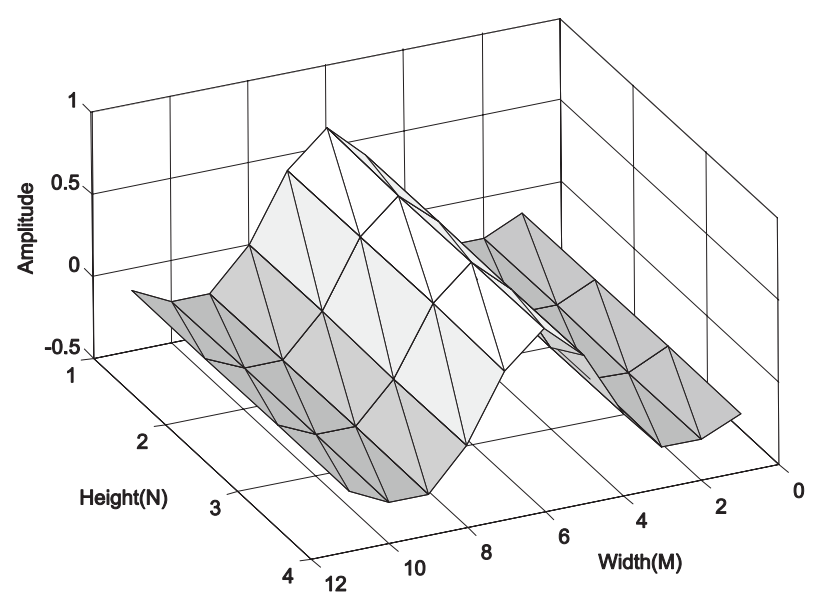

Fig. 1. Example of 2-D Mexican Hat kernel wavelet, where its width is 12 units, height 4 units and directional angle is 0 degrees.

Figure 2 exemplifies the directional wavelet transform 2-D Mexican Hat on a synthetic image (top row). The second row in Figure 2 schematically depicts the result of this transformation directions $(\delta) 0, \pi / 4$, and $\pi / 2$. 

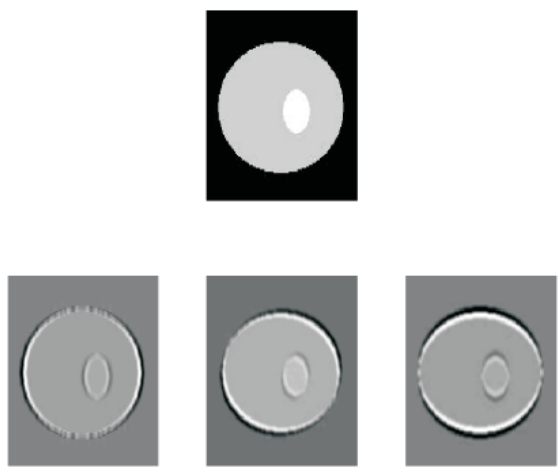

Fig. 2. A synthetic image (top) and DWT transformation results at directions $0, \pi / 4$ and $\pi / 2$ (bottom).

Figure 3, however, exemplifies the DWT on a noisy synthetic image (Gaussian noise with zero mean and 0.01 variance). The second row in Figure 3 schematically depicts these results at directions $0, \pi / 4$, and $\pi / 2$.
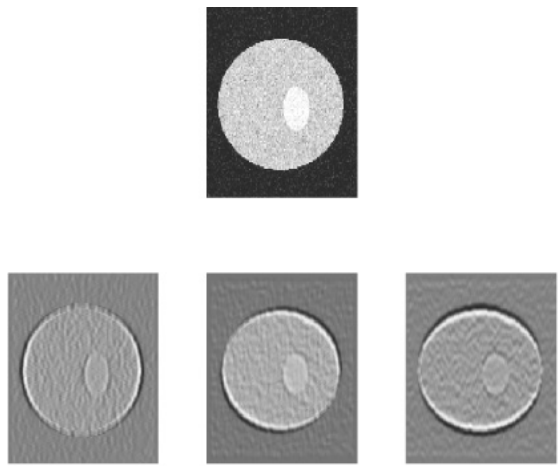

Fig. 3. A noisy synthetic image (top) and DWT transformation results at directions $0, \pi / 4$ and $\pi / 2$ (bottom).

From these examples it is clear that the kernels suppress all variations which are not parallel with their directions. Homogeneous regions result in grey colour (Figure 2, bottom row). Also, the structures, e.g. isolated singularities along the line, create cones of large amplitude coefficients between locations of these singularities. On the other hand, singularity neighbours with the same directions as kernels are emphasized as black and white pixels and significantly differ from the background. The singularity is visible as zero crossing. The more the singularity direction corresponds with the operator orientation, the more intensive is the obtained response.

However, the main benefits of directional wavelet transform are: a) signal decomposition into four-dimensional space and b) capability of detecting the singularity lines.

\section{Edge Sharpening}

Edge sharpening is the reverse process of an edge blurring or image smoothing. To be effective, it is essential that the magnitude of edge's components is larger than that of existing noise.

Simple edge sharpening scheme are done by selectively replacing a pixel point by its gradient. The computation of gradient can be done by using a three-point or the four-point gradient expression. Well known linear gradient operators are Laplacian operator, High-Pass Mask [20], Compass gradient masks [20] and also nonlinear operators, e.g. Sobel, Kirsch, and Wallis operator. In this work, we proposed the fourpoint gradient operator using least square plane interpolation.

Let $I(x, y)$ be the image in the $x y$ plane, and $z=a x+b y+c$ be a plane that fits the four neighbour points (i.e. approximation of four pixel values by a plane). An error that results when the plane $z$ is taken as the image plane is defined by

$$
\begin{aligned}
& \text { Error }^{2}= \\
& \qquad[a(x)+b(y)+c-I(x, y)]^{2} \\
& \quad+[a(x+1)+b(y)+c-I(x+1, y)]^{2} \\
& \quad+[a(x)+b(y+1)+c-I(x, y+1)]^{2} \\
& \quad+[a(x+1)+b(y+1)+c-I(x+1, y+1)]^{2}
\end{aligned}
$$

This quadratic error function is linear and can be analytically minimized by differentiating over unknown parameters $a, b$, and $c$. Setting this system of equations to 0 , the result is represented as follows

$$
\begin{gathered}
a=\frac{I(x+1, y)+I(x+1, y+1)}{2} \\
b=\frac{I(x, y+1)+I(x+1, y+1)}{2} \\
-\frac{I(x, y)+I(x+1, y)}{2} \\
c=\frac{1}{4}[3 I(x, y)+I(x+1, y)+I(x, y+1) \\
-I(x+1, y+1)-a-b]
\end{gathered}
$$


One can see that parameter $a$ gives the difference of the average of pixels in two consecutive columns. Similarly, parameter $b$ gives the difference of the average of pixels in two consecutive rows. System (5) defines the analytical model for searching local parameters of edge sharpening.

The gradient of the plane $z$ is defined by

$$
G=\left[\left(\frac{\partial I}{\partial x}\right)^{2}+\left(\frac{\partial I}{\partial y}\right)^{2}\right]^{1 / 2}
$$

or

$$
G=\left[a^{2}+b^{2}\right]^{1 / 2}
$$

which can be approximated as

$$
G=|a|+|b| \quad \text { or } \quad G=\max (|a|,|b|) .
$$

This gradient is less sensitive to noise than Laplacian operator because the averaging is done prior to differencing. On the other hand, it is still sensitive to ripples in the image $I(x, y)$. Therefore, a lot of artefacts will be introduced when the least square plane operator is used alone. Due to this sensitivity, the additional noise reduction post-processing step is necessary. The variance is estimated and considered in order to distinct between edge point and noise point. Edge point has sufficiently higher variance than a noise point. By using a window $(2 M+1) x(2 M+1)$, with $M$ chosen between 2 and 4 , the local variance can be estimated by

$$
\begin{aligned}
\sigma^{2}(x, y) & =\frac{1}{(2 M+1)^{2}} . \\
& \cdot \sum_{k=x-M}^{x+M} \sum_{j=y-M}^{y+M}[I(k, j)-m(k, j)]^{2}
\end{aligned}
$$

where

$$
m(x, y)=\frac{1}{(2 M+1)^{2}} \sum_{k=x-M}^{x+M} \sum_{j=y-M}^{y+M} I(k, j) .
$$

Afterward, the distinction between "noisy variance" and "edge variance" is evaluated. For threshold detection we decided to use Shannon entropy measure. It is a measure of information content where threshold $T$ is defined as the value which maximizes $H=H_{n}+H_{e}$. Parameters $H_{n}$ and $H_{e}$ are defined by (11)

$$
\begin{aligned}
& H_{n}(s(t))=-\sum_{i=1}^{T} p_{i} \log \left(p_{i}\right) \\
& H_{e}(s(t))=-\sum_{i=T+1}^{L} p_{i} \log \left(p_{i}\right)
\end{aligned}
$$

where the signal magnitude of value $i$ will occur with probability $\mathrm{p}_{i}$ in signal $s(t)$. Signal $s(t)$ stands for the vector of gradient variances. The vector is quantized and rescaled to the interval $[0,255]$ units. The threshold $T$ is a crucial parameter in the process of separation between edge and noisy pixels.

\section{Image Enhancement}

The image enhancement is defined as a process which reduces image noise, removes artefacts and preserves details. All these demands are partially achieved by edge sharpening and directional wavelet transform. Using edge sharpening and the method for distinction between edge and noisy pixel, the basis for image enhancement is set up. Additionally, with the directional wavelet transform the noise sensitivity of proposed technique is reduced because of multiscale and multidirectional approach.

We decided to use the Mexican Hat wavelet or Marr wavelet which is a rotation-invariant with symmetric frequency response. Its main property is the ability to present magnitude variations between adjacent intervals in a signal, which point out as the zero crossing from positive to negative or vice versa when proceeding from scale to scale in the time-scale plane. The Mexican Hat kernel (Figure 1) also preserves this property in the image-scaledirectional space.

The proposed image enhancement method consists of 7 major steps which are:

1. Directional wavelet transform is calculated with the Mexican Hat wavelet kernel at dyadic scales from 1 to $\left\lfloor\log _{2}(\min (I, J))\right\rfloor$, where $I$ and $J$ are the input image dimensions. Directions are selected between 0 and $\pi$ with $\pi / 4$ increments. The dyadic scaling sequence minimizes the time complexity of the algorithm, while the quality of singularities detection is not reduced. The result is an image-scale-directional space. 
2. A dimension reduction is implemented, so that only the maximum absolute value from the directional vector is kept for every transform space co-ordinate. It is clear that the Mexican Hat kernels which cross the singularities perpendicularly aggregate higher maximum absolute values in the singularity neighbourhoods. This dimension reduction results in an image-scale space $\mathrm{S}(\alpha, \tau 1, \tau 2)$.

3 . Gradient calculation by plane approximation (7) is done along $\tau 1$ and $\tau 2$ directions in the image-scale space $\mathrm{S}(\alpha, \tau 1, \tau 2)$. A plane, denoted as a gradient plane is obtained.

4. Noise reduction. Firstly, the detection of noisy points is done by estimating the local variance (10), where the value $\mathrm{M}$ is set to 3 pixels. Points with local variance above the threshold value $T(11)$ are denoted as singularity (e.g. edge, feature) points. All other points belong to constant, textured or noisy area. A plane, denoted as a singularity plane is obtained.

5. Corresponding singularity points from singularity planes (planes at different scales) are connected by using the same procedure as is used to connect the modulus maxima points introduced in [14]. Individual singularities are chained together to form singularity lines that indicate the positions of singularity.

6. The singularity position is determined as the position of singularity at the lowest computed scale. The plane of singularities is obtained.

7. Finally, the image enhancement is done over the nearest samples of detected singularity points. Nearest samples are all points, which were used to calculate the directional wavelet transform value at the position of singularity point. Then the enhanced point value is calculated as the intensity of original image plus the value of image scale space at the scale at which the singularity point was detected. New image intensity values are calculated as

$$
I(x, y)=I(x, y)+C \cdot S(x, y)
$$

where parameter $\mathrm{C}$ controls the image contrast. Function $\mathrm{S}$ represents the image-scale space. The result of our procedure is an image, where features, represented as the significant singularities, are enhanced.

\section{Results}

The proposed image enhancement algorithm was tested on a set of real images, as well as on synthetic images. Firstly, several tests and measurements were done over synthetic signals and images, which were corrupted by an additive zero-mean Gaussian noise. Besides the statistical evaluation, a visual assessment of clinical images was done.

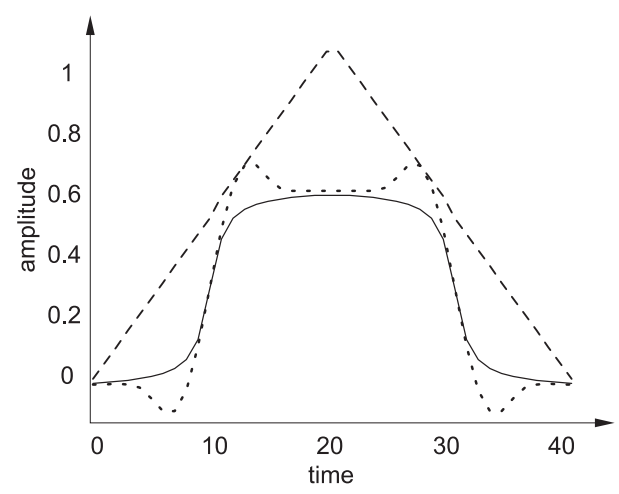

Fig. 4. 1D contrast enhancement of a synthetic signal (solid line). Dashed and dotted lines belong to histogram equalization method and our proposed method respectively $(\mathrm{C}=0.5)$.

Figure 4 depicts an 1D signal image enhancement example. Histogram equalization and our method are compared. Obviously, our method outperforms the classical histogram equalization method, because the edges are sharpened by our method, while by the histogram equalization they are more smoothed.

A quantitative measure of contrast improvement was evaluated by the Contrast Improvement Index (CII) [21]. Experimental results are averaged over 5 synthetic images. Synthetic images consisted of one homogeneous object and were corrupted by an additive Gaussian noise. The SNRs were between 3 and $50 \mathrm{~dB}$. Table 1 shows the CII in almost noiseless and noisy images. The comparison between our proposed method and histogram equalization method is done. One can find out that our method achieves higher CII indexes than histogram equalization method. On the other hand, it might be surprising that the CII of histogram equalization method is lower than original image CII. 


\section{Conclusion}

\begin{tabular}{|l|c|c|c|}
\hline $\begin{array}{l}\text { CII } \\
\backslash \text { Images }\end{array}$ & Original & $\begin{array}{l}\text { Histogram } \\
\text { equalization }\end{array}$ & $\begin{array}{l}\text { Our } \\
\text { method }\end{array}$ \\
\hline Noiseless & 0.65 & 0.53 & 0.75 \\
\hline Noisy & 0.46 & 0.51 & 0.71 \\
\hline
\end{tabular}

Table 1. CII calculated on the original image, the histogram equalized image, and the image equalized by our method.

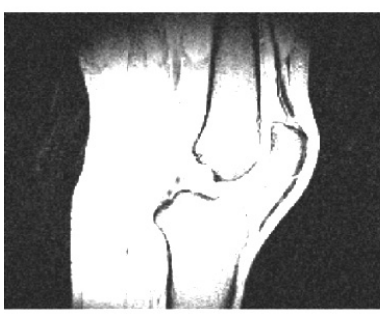

a)

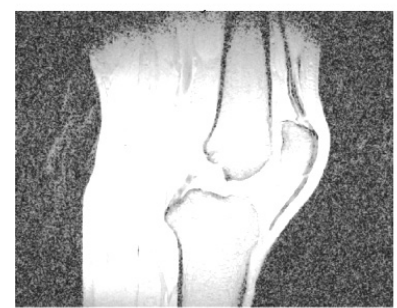

c)

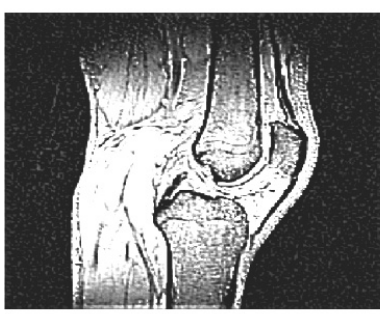

e)

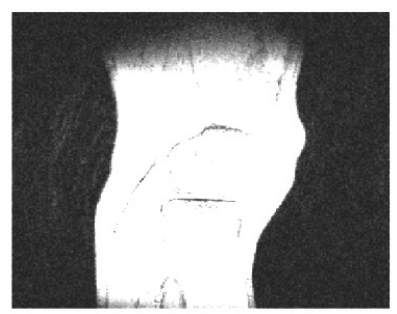

b)

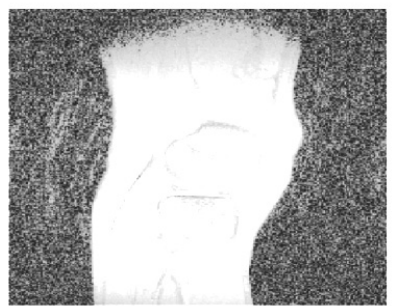

d)

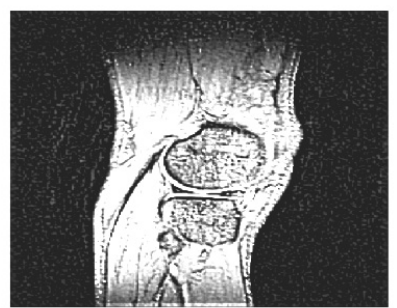

f)

Fig. 5. Original images $\left(1^{\text {st }}\right.$ row $)$, histogram equalized images $\left(2^{\text {nd }}\right.$ row $)$, images enhanced by proposed method with $\mathrm{C}=0.7$ ( $3^{\text {rd }}$ row $)$.

After synthetic image validation we performed several tests on high-quality MR-images. Figure 5 depicts results of the histogram equalization method and our method. One can see that after enhancement with both methods, significant structures like bones, ligaments, menisci, soft and hard tissues, are clearer and more visible than before. But, we might say that our method better enhances the boundaries of small and thin structures, e.g. menisci, ligaments, and various tissues like patella.

A novel image enhancement procedure was proposed in this paper. This method combines directional wavelet transform and image gradient averaging by plane modelling. By using directional wavelet transform the singularity points pointed out as zero point crossing and having high local variance. Afterwards, the plane alignment among four-points in the image-scale plane is done. Its gradient indicates the singularity significance. At the end, the image is enhanced in the neighbourhood of most significant singularities. Testing with synthetic images has showed that proposed image enhancement technique is robust, accurate, and effective in noisy images too.

\section{References}

[1] I. Pitas, A. N. Venetsanopoulos, Nonlinear Digital Filter: Principles and Applications, Kluwer Academic Publisher, Norwell, MA; 1990.

[2] D. C. Wang, A. H. Vagnucci, C. C. Li, Digital Image Enhancement, Computer Vision, Graphics, and Image Processing, 1983; Vol. 24; pp. 363-381.

[3] A. LAINE, W. HudA, Enhancement by Multiscale Nonlinear Operators, Handbook of Medical Imaging, Academic Press; 2000.

[4] A. Rosenfeld, A. KAK, Digital Picture Processing, $2^{\text {nd }}$ ed. Academic Press, New York; 1982.

[5] F. NeYCENSSAC, Contrast enhancement using the Laplacian-of-a-Gaussian filter, CVGIP: Graphical Models and Image Processing, 1985; Vol. 55: pp. 447-463.

[6] R. GORDON, R. M. RANGAYYAN, Feature enhancement of film mammograms using fixed and adaptive neighbourhoods, Applied Optics 1984; Vol. 23: pp. 560-564.

[7] A. Beghdadi, A. L. Negrate, Contrast enhancement technique based on local detection of edges, Comput. Vision Graphics Image Process 1989; Vol. 46: pp. 162-174.

[8] W. QIAN, Medical Image Enhancement with Hybrid Filters; 2000.

[9] A. F. Laine, S. Song, J. FAn, Adaptive Multiscale Processing for Contrast Enhancement, in Proceedings of SPIE 1993; Vol. 1905: pp. 521-532.

[10] B. D. JAWERTH, M. L. HiLton, T. L. HuntsBerger, Local enhancement of compressed images, J. Mathematical Imaging and Vision 1993; Vol. 3: pp. 39-49. 
[11] S. Mallat, W. L. Hwang, Singularity detection and processing with wavelets, IEEE Transactions on Information Theory 1992; Vol. 38: pp. 617-643.

[12] D. L. DonOHO, Nonlinear wavelet methods for recovery of signals, densities and spectra from indirect and noisy data, Proc. Symposia Applied Math. 1993; Vol. 47: pp. 173-205.

[13] M. N. Do, Directional Multiresolution Image Representations Ph.D. thesis, 2001.

[14] S. Mallat, A Wavelet Tour of Signal Processing 2nd Ed. San Diego: Academic Press; 1999.

[15] Y. LU, M. N. Do, A Directional Extension for Multidimensional Wavelet Transforms, IEEE Transactions on Image Processing, submitted; 2005.

[16] E. CANDÈs, Ridgelets: theory and applications, Ph.D. thesis, Department of Statistics, Stanford University; 1998.

[17] D. HERIC, D. ZAZULA, Reconstruction of object contours using directional wavelet transform, WSEAS Trans. Comput. 2005; Vol. 4, pp. 1305-1312.

[18] A. Witkin, Scale space filtering. In Proceeding of Int. Joint. Conf. Artificial Intell., Espoo, Finland 1983.

[19] C. W. Tang, H. M. Hang, A Feature-Based Robust Digital Image Watermarking Scheme, IEEE Transaction on signal processing 2003, Vol. 51: pp. 950-959.

[20] S. Bow, Pattern Recognition and Image Preprocessing, $2^{\text {nd }}$ Ed. New York, Marcel Dekker, Inc.; 2002.

[21] W. M. Morrow, R. B. Paranjape, R. M. RanGAYYAN, Region-based contrast enhancement of mammograms, IEEE Transactions on Medical Imaging 1992, Vol. 11: pp. 392-406.

Received: June, 2006 Accepted: September, 2006
Contact address:

Dusan Heric

Faculty of Electrical Engineering and Computer Scienc System Software Laboratory Smetanova 17, 2000 Maribor

Slovenia

e-mail: dusan.heric@uni-mb.si

DUSAN HERIC, born in 1978, received his BSc degree in 2002 from the University of Maribor. He currently holds a position of young researcher at the Faculty of Electrical Engineering and Computer Science, Maribor. His research field includes signal processing - wavelet transformation, image processing - segmentation, registration, cognitive vision and biomedicine. He is a member of IEEE Computer Society and of the Slovenian Pattern Recognition Society.

DR. BOZIDAR POTOCNIK, born in 1972, received his BSc degree in 2000 from the University of Maribor. Dr. Bozidar Potocnik currently holds a position of assistant professor at the Faculty of Electrical Engineering and Computer Science, Maribor. His research field includes segmentation algorithms at computer image processing, pattern recognition, biomedicine, cognitive vision, and machine learning. Dr. Bozidar Potocnik is a member of IEEE Computer Society, IEEE Society of Engineering in Medicine and Biology, and of the Slovenian Pattern Recognition Society. 